\title{
Overview of current precision clocks and future prospects
}

\author{
R. L. Beard \\ U. S. Naval Research Laboratory, Washington, D. C., U.S.A.
}

\begin{abstract}
Today's time and frequency standards range from the most sophisticated reference standards to the smallest oscillator for handheld radios. The technical requirements and technologies needed are different for the various applications but they derive from similar physical concepts. These different technologies can be categorized into four major areas, reference standards, mobile systems, man-portable (handheld) and space systems. These areas are the core areas of time and frequency standard applications and different areas of technology are needed to address them. This presentation discusses the time and frequency standards used or available for these areas.
\end{abstract}

\section{Reference Standards}

Clocks and oscillators within this area are of the type needed by reference timescale centers such as the U.S. Naval Observatory(USNO) Master Clock. This specialized area requires the most highly stable and accurate time standards that are maintained under controlled conditions. Their outputs are processed with special ensembling algorithms designed to produce an absolute reference for all systems. For example, the current suite of clocks used at USNO consists of commercial cesium beam frequency standards and hydrogen masers. These clocks are physically separated and operated in tightly controlled environment chambers. Size, weight and power are not issues pertinent for these clocks, primary emphasis is on performance, mostly in the long term.

\section{Mobile Systems}

Clocks in this area are typically crystal oscillator based devices and small atomic clock/oscillator, used for positioning, communications or internal subsystems. Although small, the requirements for mobile devices are typically not demanding or rigorous. Clock technology to produce smaller, lower power devices is slowly developing primarily through government support.

\section{Man-Portable}

Devices in this area are the most demanding in terms of size, weight and power. The most commonly used are quartz crystal oscillator devices. However, in recent years there have been several government sponsored efforts to develop extremely small atomic standards. These devices offer better accuracy and stability than crystal oscillators in an extremely small package. Although their performance exceeded that of crystal oscillator based devices, they have yet to perform as well as their larger mobile or timing center devices. 


\section{Space Qualified Atomic Standards}

Space qualified atomic clocks have been essential for the development and deployment of the Global Positioning System (GPS). GPS is the dominant user of high precision and stable space qualified atomic clocks, the only other user, are the few MILSTAR satellites containing lesser performing Rubidium standards. These clocks provide high stability for navigation performance and a large part of the development of these devices for space was to provide high stability reliably. GPS user equipment, and the timing capability resulting from the atomic clocks in the GPS system, are producing an inexpensive alternative to high precision atomic clocks for many systems. By displacing higher cost, higher performing atomic clocks, GPS User Equipment receivers or timing receivers with low quality clocks are being deployed in a wide variety of systems. Naval tactical and strategic systems have utilized hundreds of these units. Larger ships may have multiple cesium standards on board. Secondary standards such as rubidium vapor cells and crystal oscillators are being used extensively in aircraft, shipboard and man portable applications, since virtually every system has a clock or oscillator of some quality contained in it.

\section{Current Standards}

The primary frequency standard for the U.S. is the laser cooled cesium fountain at the National Institute for Standards and Technology. This type of standard is the metrological reference standard in frequency and a number of similar units are in use through the world. There are some 21 centers using cesium fountain clocks, although they are not commercially available. Each center has virtually built their our fountain clock. The performance is determined by comparison and coordination with the Bureau de Poids et Mesure. For other than timing centers, such as telecommunications centers, the most prevalent standard is the commercial cesium standard. Second is the active hydrogen maser that is in limited commercial availability. These devices are expensive with the hydrogen maser being about an order of magnitude more expensive.

The space qualified atomic clocks in the GPS operational satellites have stability requirements ranging from $2 \times 10^{-13} /$ day for cesium and $1 \times 10^{-14} /$ day for the rubidium on the later satellites. On-orbit performance has provided better than expected stabilities. The use of hydrogen masers for the ground stations and eventually in spacecraft was considered even before the beginning of the GPS Program. Efforts at that time were based on adapting the active hydrogen maser design initially developed by the Smithsonian Astrophysical Observatory. Their Gravity Probe One unit, built for a NASA sponsored relativity experiment, was launched in the mid-1970's and demonstrated potential for operation in orbiting spacecraft. To reduce the size of the active hydrogen maser a compact passive physics unit design was developed by U.S. Naval Research Laboratory for GPS. The final selected approach was the Hughes Q-enhanced design with a small magnetron cavity. This maser design approach reduced the overall unit size roughly to that of a GPS space qualified cesium clock. The European development of a navigation satellite system, to be known as GALILEO, has also developed rubidium and hydrogen maser clocks for spacecraft. The latest technology satellite, known a Giove B, has a space qualified hydrogen maser on-board that has thus far shown very good results.

\section{Potential Future Clocks}

Microwave standards are a mature technology and have good potential for further significant improvements. For instance, a "juggling" rubidium fountain clock that launches 
multiple "balls" of atoms in rapid succession could greatly improve the Signal to Noise Ratio (SNR) and result in short term fractional frequency stability in the high $10^{-15^{\prime} \mathrm{s}}$ at one second while still maintaining excellent long term systematics well below $10^{-16}$. This stability requires an local oscillator (LO) with better performance than an Ovenized Crystal Oscillator (OCXO). The Time and Frequency Group at the Jet Propulsion Lab (JPL) in Pasadena has built a cryo-cooled sapphire-loaded ruby oscillator that achieves $3 \times 10^{-15}$ performance from 1 to 1000 seconds, thus meeting the local oscillator requirements for an advanced fountain. It is possible that further refinements to the fountain concept could bring that device into the low $10^{-15^{\prime} \text { s }}$ at a second. Laser-cooled neutral atom microwave standards based on rubidium have been under development by USNO, and they are in the process of incorporating them into their operations.

Laser-cooled microwave ion standards are expected to have an exceptional long term systematic noise floor. It is likely that the main limitation will be magnetic field sensitivity, which is largely an engineering problem of providing good shielding while still maintaining good optical access. However, while the systematic floor is likely to be in the low $10^{-17^{\prime} \mathrm{s}}$, the short term stability is probably limited to the low $10^{-13^{\prime} \mathrm{s}}$ due to the low SNR inherent in a device with only a few ions. As a result, a microwave laser-cooled ion trap device is unlikely to meet the stated goals.

Buffer-gas-cooled ion standards have already demonstrated a stability of $3 \times 10^{-14}$ at 1 second. These devices have large signals (many ions), but only a moderate SNR due to large background signals. A factor of 3-10 improvement in SNR could be achieved with better detection schemes to reduce background. This almost certainly means using lasers instead of lamps, as is the current practice. One of these ion standards coupled with an advanced LO (such as the cryo-cooled LO already discussed) could get close to the short term stability goal, but the systematic floor is unlikely to be below $10^{-16}$ (larger numbers of ions at higher temperatures means both exposure to higher rf fields and larger Doppler shifts). Nevertheless, this type of approach should not be dismissed too quickly, since this frequency stability still allows several ps timing stability at one day. The buffer-gas-cooled ion standard with laser interrogation would require the fewest technological advances and would be the simplest to implement.

The JPL Time and Frequency Group has developed a new technology standard known as the Linear Ion Trap Standard. Operational versions of these units are being deployed in the NASA Deep Space Network as replacements for the large active hydrogen masers currently in use. A spacecraft version of these units is being investigated and offers the potential of very small size and power for potentially high stability. The physics package has been shown to be capable of small design, but since it is a passive device, a high quality local oscillator is needed to gain the full potential of these devices. The potential performance gain using a modest performance local oscillator and the adaptability to digital implementation of the electronics could be a major step in space qualified atomic clock technology.

The next step in atomic clock evolution is to move from microwave "clock" frequencies to optical frequencies. With frequencies measured in the $10^{15} \mathrm{~Hz}$ range instead of $10^{10}$ $\mathrm{Hz}$, optical clocks have a potentially huge gain in $Q$ (the ratio of the oscillator frequency to the uncertainty in that frequency). Since short-term stability is inversely proportional to $Q$, it too improves. An ion trap clock based on an optical transition then combines very good short-term stability due to the high $Q$ of the optical transition with an exceptionally low systematic noise floor.

There are two technologies that are critical to optical clock progress. The first is Octavewide Optical Comb Generation (OOCG). This is the phase-coherent spanning of a factor of two in optical frequencies by a frequency "comb" with radio frequency (rf) (100 to 
$1000 \mathrm{MHz}$ ) spacing. The OOCG makes it possible to link optical frequencies coherently down to rf frequencies, where timing information is usually generated, transferred and analyzed. The first successful OOCGs have been demonstrated, but significant research needs to be performed to map out their characteristics and capabilities. This is a huge step for optical clocks, since previous chains linking optical to rf frequencies required man-years of highly skilled work to build and maintain. With advent of OOCGs the amount of work has been reduced by several orders of magnitude. The second critical technology is laser frequency stabilization. To take full advantage of the optical line $Q$, the "clock" laser, which is now the LO, must have a frequency uncertainty on the order of $1 \mathrm{~Hz}$ or less. This is difficult to achieve but offers great potential for future development of clock technology. 